\title{
Pregnancy-Induced Functional Class Modifications in Patients with Congenital Heart Disease
}

Daliento L*, Pomiato E, Mazzotti E, Bauce B, Spadotto V and Rizzoli G

Department of Cardiac, Thoracic and Vascular Sciences, University of Padova, Italy

*Corresponding author: Daliento L, Department of Cardiac, Thoracic and Vascular Sciences, University of Padova Medical School, Via Giustiniani, 2, 35121- Padova (PD), Italy

Received: April 10, 2017; Accepted: May 23, 2017; Published: May 31, 2017

\begin{abstract}
Background: Pregnancy represents a raising issue in women with Congenital Heart Disease (CHD), but its effect on functional status is not well established. The aim of our study was to assess the probability of modification of functional status due to pregnancy in $\mathrm{CHD}$, thus improving preconceptional counselling.
\end{abstract}

Methods: Data on maternal NYHA functional class and Ability Index before, during and after pregnancy in 127 pregnancies in 95 women (mean age $31 \pm 5$ years) with CHD, collected between 2008 and 2013 at Padua University Hospital Clinic were recorded (total visits 498), together with echocardiographic parameters.

Results: Before pregnancy, 93 patients were in NYHA Class I and 9 in NYHA class II; during pregnancy 83 were in class I and 14 in class II; during puerperium, 81 were in class I and 15 in class II; at 6-month follow-up, 82 were in class I and 19 in class II. Worsening of NYHA class from baseline to 6-months after pregnancy was significantly associated to baseline NYHA class $>1$ (OR $127 p<0.0001$ ), number of pregnancies (OR 1.014, $p=0.03$ ), history of palliative operation prior to correction (OR 2.4, $p<0.0001$ ), corrective surgery (OR 3.47 , $\mathrm{p}<0.0001)$ and complex CHD (OR 5.3, p<0.0001). At multivariate analysis, baseline NYHA, and complex CHD remained independent predictors of NYHA class deterioration. Cardiac ultrasound parameters showed the same trend as NYHA.

Conclusions: In-women with CHD the probability of functional class worsening during and after pregnancy adjusted for concomitant risk factors is significantly related to baseline NYHA class and complexity of CHD.

Keywords: GUCH, pregnancy, congenital heart, NYHA, echocardiography

\section{Abbreviations}

ASD: Atrial Septal Defect; CHD: Congenital Heart Disease; CL: Confidence Limit; FAC: Fractional Area Change; GUCH: Grown-Up Congenital Heart; LMP: Last Menstrual Period; LVEF: Left/Systemic Ventricle Ejection Fraction; PDA: Patent Ductus Arteriosus; PFO: Patent Foramen Ovale With Positive Bubbles Test (PFO); RV: Right Ventricle; Rvdil: Right Ventricle Dilatation; RVEDA: Right Ventricle End-Diastolic Area; Rvfunct: Right Ventricle Function; SPAP: Systolic Pulmonary Artery Pressure; TAPSE: Tricuspid Annular Plane Systolic Excursion; TGA: Transposition Of Great Arteries; TOF: Tetralogy Of Fallot; VSD: Ventricular Septal Defect.

\section{Introduction}

Progress in prenatal screening, surgical techniques and neonatal management of Congenital Heart Disease (CHD) led to an increasing number of Grown up Congenital Heart (GUCH) patients. Therefore, new psychological and clinical issues are raising [1], among them an increasing number of women with $\mathrm{CHD}$ want to become pregnant [2].

Fertility in women with CHD is generally preserved [3], but other factors can influence pregnancy outcome and maternal and foetal well-being [4-6].
Many studies have tried to develop maternal and foetal risk scores in this setting $[7,8]$, but simpler predictors are needed to provide an effective counselling. The aim of this study was to evaluate the degree of maternal functional worsening and the foetal outcome in large number of pregnancies among CHD women.

\section{Material and Methods}

\section{Study design}

The study retrospectively analysed 127 consecutive pregnancies occurred in GUCH women between 2008 and 2013, followed by the Department of Cardiac, Thoracic, and Vascular Sciences and/or the Department of Maternal and Pediatric Health of the Padua University Hospital. Women were divided into two categories: patients with simple CHD (native or surgically repaired) included Atrial Septal Defect (ASD), patent foramen ovale with Positive Bubbles Test (PFO), Ventricular Septal Defect (VSD), patent ductus arteriosus, cor triatriatum, coronary fistula, bicuspid aortic valve, aortic coarctation, pulmonary stenosis, partial anomalous pulmonary venous return and congenital atrioventricular block. Patients with complex CHD (native or surgically corrected) encompassed Tetralogy of Fallot (TOF), atrioventricular septal defect, Transposition of Great Arteries (TGA), double-outlet left ventricle, double-outlet right ventricle, Ebstein's 
Table 1: Type of congenital heart disease and related pregnancy outcome.

\begin{tabular}{|c|c|c|c|c|}
\hline & $\begin{array}{c}\text { Pregnancy } \\
\mathbf{n}=\text { value (\%) }\end{array}$ & $\begin{array}{c}\text { Delivery } \\
\mathbf{n}=\text { value } \\
\mathbf{( \% )}\end{array}$ & $\begin{array}{c}\text { Miscarriage } \\
\mathbf{n}=\text { value (\%) }\end{array}$ & $\begin{array}{c}\text { Voluntary } \\
\text { abortion } \\
\mathbf{n}=\text { value (\%) }\end{array}$ \\
\hline $\begin{array}{c}\text { simple } \\
\text { operated } \\
\text { CHD }\end{array}$ & $25(20)$ & $18(18)$ & $6(40)$ & $1(10)$ \\
\hline $\begin{array}{c}\text { simple not } \\
\text { operated } \\
\text { CHD }\end{array}$ & $38(32)$ & $32(31)$ & $2(13)$ & $4(40)$ \\
\hline $\begin{array}{c}\text { complex } \\
\text { operated } \\
\text { CHD }\end{array}$ & $61(48)$ & $51^{*}(50)$ & $7(47)$ & $3^{\star *}(30)$ \\
\hline $\begin{array}{c}\text { complex not } \\
\text { operated } \\
\text { CHD }\end{array}$ & $3(2)$ & $1(1)$ & $0(0)$ & $2(20)$ \\
\hline TOTAL & $127(100)$ & $102(100)$ & $15(100)$ & $10(100)$ \\
\hline
\end{tabular}

CHD: Congenital Heart Disease

${ }^{*} 50$ live birth; 1 stillborn at 40 gestational weeks; " $2<90^{\circ}$ gestational day; $1>$ $90^{\circ}$ gestational days.

anomaly and Marfan syndrome.

NYHA functional class and Ability Index [9] were found in medical records. We evaluated them in all pregnancies: before gestation, during it, 1 and 6 months after delivery; thereafter, we continued data collection up to 16 years after delivery, but some patients drop-out.

We collected echocardiographic exams performed before, during and after pregnancy and evaluated them following the most recent echocardiographic guidelines [10].

We also evaluated pregnancy outcome, obstetric complications, time and mode of delivery, offspring outcome, Apgar score [11] and recurrence of CHD.

\section{Statistical analysis}

Continuous data were summarised with means (standard deviation) when normally or medians (interquartile range) when non-normally distributed. Relationship between predictors and events was assessed with univariate and multivariate analysis. The hazard of miscarriage and abortion was empirically calculated and parametrically modelled according to Royston Flexible parametric survival models [12].

NYHA class during pregnancy, puerperium and post-puerperium was analysed with a multilevel longitudinal and structural mixed model with an ordered logit link (Stata Meologit procedure) to exclude confounding effects due to random variability among patients and among consecutive pregnancies in a single patient.

Time related echocardiographic changes were analyzed with a non-parametric test for trend across ordered group (Stata Nptrend procedure).

A value of $\mathrm{p}<0.05$ was considered significant. Statistics were analyzed with STATA version 12 (Stata Corp 4906 Texas USA).

\section{Ethical approval}

The study protocol conforms to the ethical guidelines of the 1975 Declaration of Helsinki and was approved a priori by our local Ethical Committee (Comitato Etico Provinciale Padova). As statistical analysis was performed on de-identified historical data, the institutional review board waived the need for written informed

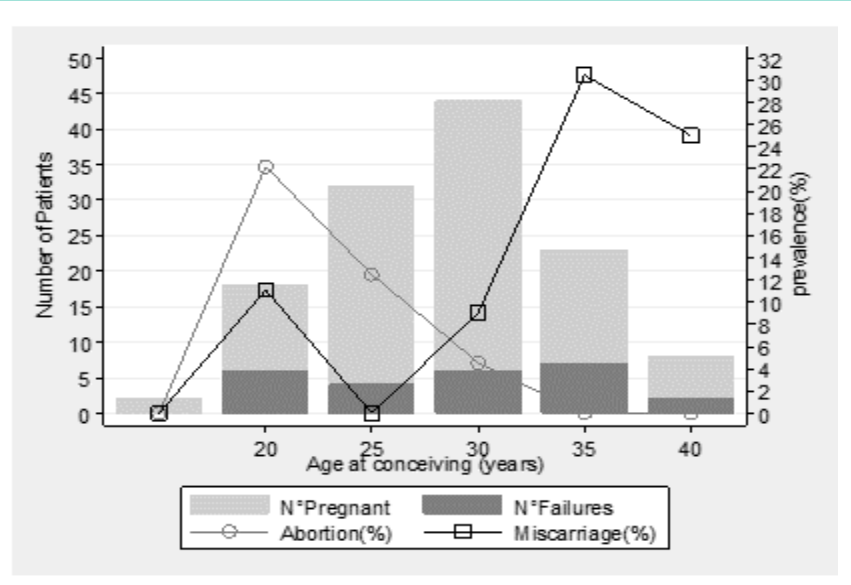

Figure 1: Proportion of voluntary abortions and miscarriages according to maternal age.

Number of patients with pregnancies (light grey) and pregnancy failures (dark grey) and prevalence of either miscarriage (squares) or abortion (circles) are plotted according to classes of maternal age.

consent from the participants.

\section{Results}

\section{Population}

The study analysed 127 consecutive pregnancies occurred between 2008 and 2013 among 95 women with CHD. 63 pregnancies (50\%) occurred in women with simple CHD (25 in corrected CHD, 38 in native CHD) and 64 (50\%) in women with complex CHD (61 in corrected $\mathrm{CHD}, 3$ in native $\mathrm{CHD}$ ). The median maternal age at the time of conception was 31 years ( $\min 26, \max -33$ yrs). Multiple pregnancies were treated as independent events.

\section{Pregnancy outcome and risk of adverse events}

125 pregnancies $(98 \%)$ had natural conception, while two (2\%) required medically assisted procreation techniques. In 25 cases (20\%) pregnancies did not end in delivery, as there were 15 miscarriages and 10 voluntary abortions (Table 1). Miscarriages occurred in 8 out 63 pregnancies of simple CHD patients (13\%) and in 7 out 64 pregnancies in complex CHD patients $(11 \%),(\mathrm{p}=0.78)$. The incidence of miscarriages was higher (15\%) among women $<30$ years old compared to those $\geq 30$ years old ( $3 \% ; p=0.02$ ) (Figure 1 ).

Delivery occurred in 102 pregnancies, by mean of caesarean section in $70 \%$, after a median of 39.3 weeks (37.7-40 weeks). Four (4\%) deliveries occurred before 32 weeks of gestation. There was no difference in mean gestation duration between patients with simple versus complex CHD, but 3 out of 4 early deliveries occurred in surgically corrected CHD.

We did not observe any maternal dead during or after pregnancy.

Mean Apgar score at $1^{\text {st }}$ and $5^{\text {th }}$ minute after birth was $8.7 \pm 1.4$ (range 2-10) and 9.7 \pm 0.6 (range 7-10). We observed one stillbirth at $40^{\text {th }}$ gestational week and one very early preterm baby ( 26 weeks) who suffered severe perinatal complications (bronco pulmonary dysplasia, haemorrhagic enterocolitis and cerebral damage). All remaining 101 babies did not suffer of perinatal complications.

Congenital heart defects were observed in 7 newborns (6.9\%) but 
diseases were different from those of the mothers.

\section{Maternal NYHA class changes}

Among the 102 pregnancies with delivery, 93 patients (91\%) were in NYHA functional Class I before the gestation and 9 (9\%) in Class II, while no patients were in NYHA Class III or IV. In 83 out 93 pregnancies (89\%), mothers with baseline NYHA Class I had no clinical worsening, while 10 (11\%) progressed to Class II during pregnancy and 3 additional patients worsened to Class II at 1-month follow-up.

At the end of follow-up, 80/93 (86\%) of patients with baseline NYHA class I remained in Class I 6 months after pregnancies while 13 worsened to Class II (14\%). Among the 9 pregnancies with baseline Class II, in 5 cases the patients worsened to Class III. Of these, one returned to Class II at 6-month follow-up. Overall, worsening of functional status occurred in 18 out 102 successful pregnancies (18\%) and it has been related with pre-term delivery. 3 of these patients had native simple CHD (17\%): 2 had ASD (one discovered during pregnancy); the third had a known bicuspid aortic valve and experienced progressive heart failure soon after delivery; the other 15 pregnancies (83\%) occurred in surgically corrected CHD: 10 TOF, 1 VSD with pulmonary stenosis; 1 complex TGA; 1 coarctation of the aorta associated with aortic stenosis; 1 double-outlet right ventricle in ventricular D-loop and situs ambiguous; 1 double-outlet left ventricle with severe pulmonary stenosis, straddling and overriding of right atrio-ventricular valve and large VSD.

2 patients out of 10 with native PFO (20\%) experienced a Transitory Ischaemic Attack (TIA) during pregnancy and the first month after the delivery.

No surgical cardiac procedures were performed during pregnancy. 11 women (9\%) underwent cardiac surgical or interventional procedure after gestation: in 4 patients $(36 \%)$ with ASD or PFO, the defect was closed percutaneously; 3 (28\%) patients affected by TOF underwent pulmonary valve replacement; in 1 (9\%) patient with bicuspid aortic valve and VSD, aortic valve replacement was performed; 1 (9\%) woman with bicuspid developed aortic aneurism and underwent cardiac surgery; 1 (9\%) patient with coarctation of the aorta developed atrial fibrillation.

Univariate analysis of the determinants significantly associated with NYHA Class worsening identified the baseline NYHA Class II with an OR of $127(\mathrm{p}<0.0001)$, complex CHD (OR 5.3, $\mathrm{p}<0.0001)$, corrective surgery (OR 3.47, $\mathrm{p}<0.0001$ ), a history of palliative operation prior to correction (OR 2.4, $\mathrm{p}<0.0001)$, multiple pregnancies (OR 1.014, $\mathrm{p}=0.03$ ). Pregnancy failures (miscarriage or abortion) were not significant (OR 1.23, $\mathrm{p}=0.6)$. Multivariate analysis showed a significant independent effect of baseline NYHA and presence of complex CHD on the probability of NYHA Class worsening (Table 2).

\section{Maternal ability index changes}

Evaluation of the Ability Index showed a significant deterioration $(\mathrm{p}=0.0003)$ from a pre-gestational mean of 1.02 to a mean of 1.29 at follow-up. In women with native simple CHD, the average Ability Index increased to $1.125 \pm 0.34(\mathrm{p}=0.04)$ in pregnancy and to $1.19 \pm 0$ $.47(\mathrm{p}=0.026)$ in puerperium but returned toward baseline $1.09 \pm 0.3$ $(\mathrm{p}=0.09)$ at later follow-up.

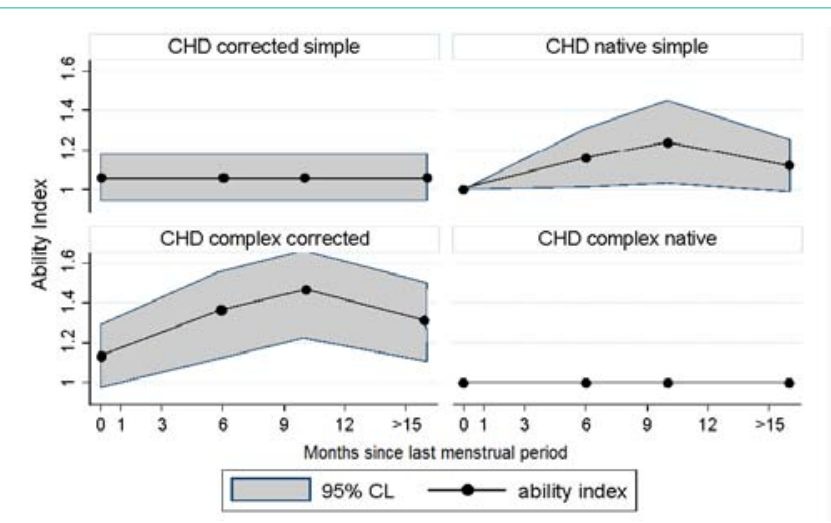

Figure 2: Trend of Ability Index across time since last menstrual period according to CHD type.

Data are presented as mean NYHA value and 95\% confidence interval. CHD: Congenital Heart Disease; CL: Confidence Limit.

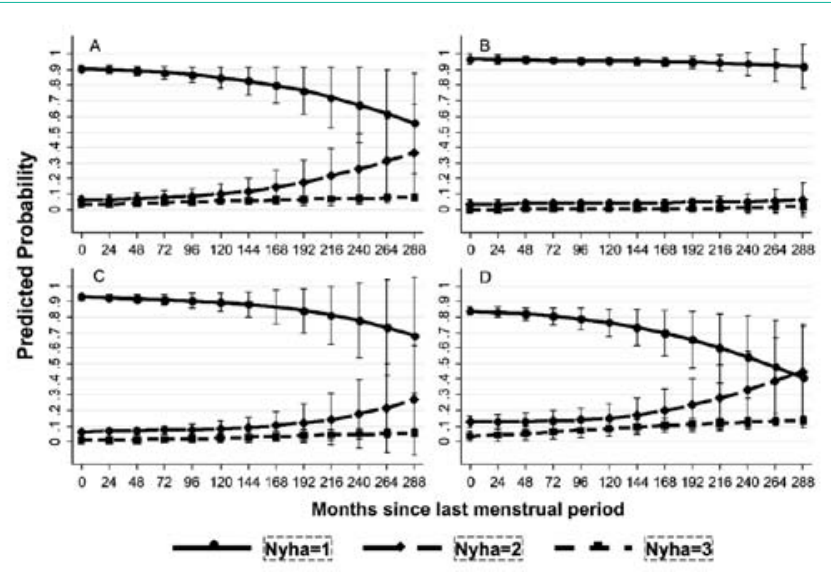

Figure 3: Probability of staying stable in the same NYHA class.

Data are presented as mean probability and $95 \%$ confidence interval in: (A) overall women with $\mathrm{CHD}$; $(\mathrm{B})$ women with simple corrected $\mathrm{CHD}$; $(\mathrm{C})$ women with native simple CHD; (D) women with complex CHD. CHD: Congenital Heart Disease.

Among the 50 surgically treated patients with complex CHD, baseline Ability Index was $1.14 \pm 0.40$ and increased to $1.39 \pm$ $0.57(\mathrm{p}=0.014)$ during pregnancy and up to $1.45 \pm 0.58(\mathrm{p}=0.003)$ at 1-month follow-up. At 6-month follow-up, recovery was still incomplete, although mean Ability index do not statistically differ from baseline $(1.38 \pm 0.53, \mathrm{p}=0.09)$. The single native complex patient persisted with an Ability Index of 1 throughout pregnancy and follow-up (Figure 2).

Multivariate analysis showed that Ability Index change was related to baseline asthenia (OR 6, $\mathrm{p}=0.005)$ and gestational dyspnoea (OR 4, $\mathrm{p}=0.05$ ); while presence of multiple deliveries showed a borderline statistical significance (OR 1.9, p=0.08).

\section{Prediction of NYHA change after pregnancy}

According to the factors of Table 2 we can predict the probability of being in the stated NYHA class during pregnancy and in the short and mid-term after delivery (Figure 3 ).

\section{Echocardiographic parameters}

Ventricular volumes, ejection fraction and valvular parameters 
Table 2: Predictors of NYHA Class worsening at multivariate analysis.

\begin{tabular}{|c|c|c|c|c|}
\hline & Coef. & Std. Err. & p-value & {$[95 \% \mathrm{Cl}]$} \\
\hline $\begin{array}{c}\text { baseline NYHA (II } \\
\text { vs. I) }\end{array}$ & 7.18 & 1.011 & 0.0001 & $5.21-9.17$ \\
\hline $\begin{array}{c}\text { CHD simple not } \\
\text { operated }\end{array}$ & 2.04 & 1.586 & 0.198 & sss \\
\hline CHD complex & 3.27 & 1.510 & 0.030 & $0.31-6.23$ \\
\hline $\begin{array}{c}\text { Change of CHF } \\
\text { therapy }\end{array}$ & 3.12 & 1.117 & 0.005 & $0.93-5.31$ \\
\hline Follow-up years & 0.15 & 0.035 & 0.000 & $0.09-0.22$ \\
\hline
\end{tabular}

CHD: Congenital Heart Disease; CHF: Congestive Heart Failure; Cl: Confidence Interval; Coef: Regression coefficient; Std. Err.: Standard error; vs: Versus

Table 3: Overall left (for biventricular hearts)/systemic (for univentricular hearts) ventricle echocardiographic parameters.

\begin{tabular}{|c|c|c|c|c|}
\hline & LVEDVEX & LVEDVI & LVESVI & LVEF \\
\hline Bas & 3 & 18 & 27. & 61.2 \\
\hline & & & & 20 \\
\hline $7^{\text {th }}-9^{\text {th }} n$ & 119.6 & 65.3 & & 20 \\
\hline $10^{\text {th }}-12^{\text {th }}$ & $106.6(36.0) 19$ & 63.7 (16.8) 38 & 29.0 (20.7) 20 & $61.1(6.1) 41$ \\
\hline $13^{\text {th }}-2$ & 6 & 8 & & .5) 9 \\
\hline $25^{\text {th }}-48^{\text {th }}$ & 101.2 & 62.3 & 15 & $57.3(6.3) 18$ \\
\hline $49^{\text {th }}-72^{\text {th }} \mathrm{mth}$ & $135.8(43.3) 4$ & $67.2(22.3) 11$ & $35.3(24.1) 4$ & $62.2(7.0) 13$ \\
\hline $73^{\text {th }}-12$ & $93.6(19.1) 5$ & $57 . \varepsilon$ & $27(10.8) 5$ & $53.8(8.0) 5$ \\
\hline $121^{\text {st }}-240^{\text {th }} \mathrm{mth}$ & $105.9(21.2) 7$ & $63.6(12.3) 7$ & $29.6(10.4) 7$ & $53.7(9.1) 7$ \\
\hline $241^{\text {st }}-360^{\text {th }} \mathrm{mth}$ & $133(0) 1$ & $80(0) 1$ & $38(0) 1$ & $52(0) 1$ \\
\hline
\end{tabular}

Results are expressed by mean, (SD value) and number of observations.

Del: Delivery; LVEDVEX: Left Ventricle End-Diastolic Volume $(\mathrm{mL})$; LVEDVI: Left Ventricle End-Diastolic Volume Indexed $\left(\mathrm{mL} / \mathrm{m}^{2}\right)$; LVEF left ventricle ejection fraction (\%); LVESVI left ventricle end-systolic volume indexed $\left(\mathrm{mL} / \mathrm{m}^{2}\right)$; $\mathrm{mth}$ : month from last menstrual period.

for all CHD are shown in Table 3 and 4 (Table 4, see Supplementary Information); clinical and echocardiographic data are summarized in (Table 5) for simple CHD and in Table 6 for complex CHD. Echocardiographic left and right ventricle parameters are plotted in Figure 4 and 5 respectively. Right ventricular parameters were not measured in two univentricular heart patients.
Data show statistically significant worsening from baseline for Left/systemic Ventricle Ejection Fraction (LVEF) $(p=0.001)$, for Right Ventricle (RV) Fractional Area Change (FAC) $(\mathrm{p}=0.005)$, RV function (RVFunct) $(p=0.003), R V$ Dilatation (RVDil) $(p=0.01)$, aortic insufficiency $(p=0.02)$, tricuspid insufficiency $(p=0.006)$, pulmonary insufficiency $(\mathrm{p}=0.02)$, Systolic Pulmonary Artery Pressure (SPAP) $(p=0.02)$. A trend towards worsening was observed in left ventricle end-diastolic volume indexed $(p=0.06)$ and extended $(\mathrm{p}=0.07)$, end-systolic volume indexed $(\mathrm{p}=0.07)$ and aortic stenosis $(\mathrm{p}=0.081)$.

Changes in RV End-Diastolic Area (RVEDA) ( $p=0.11)$, Tricuspid Annular Plane Systolic Excursion (TAPSE) $(\mathrm{p}=0.22)$ and in other valvular insufficiency or stenosis did not reach statistical significance.

\section{Discussion}

The number of GUCH women who desire to have a baby is increasing and pre-gestational counselling is paramount: important and validated studies as CARPREG [7], ZAHARA [5,13] and WHO classification [14] identified the major risk factors associated with cardiac events during pregnancy. Pregnancy effects on foetal growth and clinical status in women with congenital heart disease have already been investigated $[15,16]$.

A previous study from our group showed how pregnancy could increase the incidence of arrhythmias in patients operated on for Tetralogy of Fallot [17]. In the present study, besides a traditional functional index such as NYHA class, we investigated Ability Index that fits better to GUCH population as referred to the ability to perform everyday life activities [18]. As shown by empiric trends (Figure 3), there is evidence of time related worsening of NYHA class: independent risk factors analysis and model derived predictions shows that this trend is not only limited to mothers with severe CHD; moreover, mothers with native simple CHD, even if silent, can be at risk during pregnancy.

The trend of functional class is the same both in simple and in complex $\mathrm{CHD}$ and in both categories its worsening can lead to

Table 4: Overall right ventricle and valvular echocardiographic parameters in biventricular physiology hearts.

\begin{tabular}{|c|c|c|c|c|c|c|c|c|c|c|c|}
\hline & RVFunct & RVDil & MS & MR & Aos & AoR & PS & PR & TS & TR & PHyp \\
\hline Baseline & $0.1(0.3) 27$ & $0.4(0.9) 27$ & $0(0) 28$ & $0.4(0.5) 28$ & $0.3(0.9) 28$ & $0.3(0.5) 28$ & $0.2(0.6) 28$ & $0.2(0.5) 28$ & $\begin{array}{c}0.03(0.2) \\
28\end{array}$ & $0.6(0.5) 28$ & $0.1(0.4) 28$ \\
\hline $1^{\text {st }}-6^{\text {th }}$ mth & $0(0) 16$ & $1.2(1.9) 17$ & $0(0) 17$ & $0.4(0.6) 18$ & $0.2(1.0) 17$ & $0.3(0.5) 17$ & $0.2(0.7) 17$ & $0.3(0.8) 17$ & $0(0) 17$ & $0.3(0.5) 18$ & $0(0) 14$ \\
\hline $7^{\text {th }}-9^{\text {th }}$ mth (del) & $0.1(0.3) 23$ & $1.3(2.0) 22$ & $0(0) 23$ & $0.6(0.5) 24$ & $0.2(0.9) 23$ & $0.2(0.3) 23$ & $0.2(0.6) 23$ & $0.4(0.7) 24$ & $0(0) 23$ & $0.5(0.6) 23$ & $0.1(0.3) 21$ \\
\hline $10^{\text {th }}-12^{\text {th }} \mathrm{mth}$ & $0.1(0.2) 40$ & $2.7(2.3) 40$ & $0(0) 39$ & $0.5(0.4) 39$ & $0.1(0.6) 39$ & $0.4(0.4) 39$ & $0.3(0.7) 38$ & $0.9(1.1) 38$ & $0(0) 39$ & $1.0(0.7) 39$ & $0.3(0.5) 38$ \\
\hline $13^{\text {th }}-24^{\text {th }} \mathrm{mth}$ & $0.2(0.5) 25$ & $3.2(1.9) 25$ & $0(0) 25$ & $0.46(0.3) 25$ & $0.12(0.6) 25$ & $0.54(0.5) 25$ & $0.48(0.8) 25$ & $0.78(0.8) 25$ & $0(0) 25$ & $1.0(0.6) 25$ & $0.16(0.4) 25$ \\
\hline $25^{\text {th }}-48^{\text {th }} \mathrm{mth}$ & $0.5(0.7) 17$ & $4.1(2.0) 16$ & $0(0) 17$ & $0.6(0.2) 17$ & $0.1(0.3) 16$ & $0.6(0.5) 16$ & $0.4(0.7) 17$ & $2.3(1.6) 17$ & $0(0) 17$ & $1.1(0.7) 17$ & $0.4(0.5) 17$ \\
\hline $49^{\text {th }}-72^{\text {th }} \mathrm{mth}$ & $0.8(0.9) 8$ & $4(1.6) 8$ & $0(0) 8$ & $0.8(0.4) 8$ & $0(0) 8$ & $0.6(0.6) 8$ & $0.4(0.7) 8$ & $2.6(1.7) 8$ & $0(0) 8$ & $1.3(0.8) 8$ & $0.4(0.5) 8$ \\
\hline $73^{\text {th }}-120^{\text {th }} \mathrm{mth}$ & $0.8(0.8) 5$ & $3.4(2.3) 5$ & $0(0) 5$ & $0.9(0.2) 5$ & $0(0) 5$ & $1.3(0.7) 5$ & $0.2(0.4) 5$ & $1.6(1.1) 5$ & $0(0) 5$ & $1.8(0.7) 5$ & $0.6(0.5) 5$ \\
\hline $121^{\text {st }}-240^{\text {th }} \mathrm{mth}$ & $1.5(0.6) 4$ & $4(2) 4$ & $0(0) 4$ & $0.6(0.3) 4$ & $0(0) 4$ & $0.8(0.5) 4$ & $0.25(0.5) 4$ & $2.1(1) 4$. & $0(0) 4$ & $2.3(0.9) 4$ & $0.7(0.6) 3$ \\
\hline $241^{\text {st }}-360^{\text {th }} \mathrm{mth}$ & $1(0) 2$ & $2(1.4) 2$ & $0(0) 2$ & $0.5(0.7) 2$ & $0(0) 2$ & $1.3(0.4) 2$ & $0(0) 2$ & $0.5(0.7) 2$ & $0(0) 2$ & $1(0) 2$ & $0(0) 2$ \\
\hline
\end{tabular}

AoR: Aortic Valvular Regurgitation ( $0=$ absent; 1 = mild; $2=$ moderate; 3 = severe); AoS: Aortic Valvular Stenosis $(0=$ absent; $1=$ mild; $2=$ moderate; 3 = severe); del: delivery; MR: Mitral Regurgitation ( $0=$ absent; 1 = mild; $2=$ moderate; $3=$ severe); MS: Mitral Stenosis $(0=$ absent; $1=$ mild; $2=$ moderate; $3=$ severe); PHyp: Pulmonary Hypertension ( $0=$ absent; 1 = present); Mth: Month from last menstrual period; PR: Pulmonary Regurgitation $(0=$ absent; $1=$ mild; $2=$ moderate; $3=$ severe); PS: Pulmonary Stenosis $\left(0=\right.$ absent; 1 = mild; $2=$ moderate; 3 = severe); RVDil: Right Ventricle Dilatation (0 if RVEDA $8-20 \mathrm{~cm}^{2} ; 1$ if RVEDA $21-23 \mathrm{~cm}^{2} ; 2$ if RVEDA $24-26$ $\mathrm{cm}^{2} ; 3$ if RVEDA $27-29 \mathrm{~cm}^{2} ; 4$ ifRVEDA > $\left.29 \mathrm{~cm}^{2}\right)$; RVEDA: Right Ventricle End-Diastolic Area $\left(\mathrm{cm}^{2}\right)$; RVFunct: Right Ventricle Function $(0=$ normal; 1 = mildly impaired; 2= moderately impaired; 3 = severely impaired); TR: Tricuspid Regurgitation; TS: Tricuspid Stenosis. 
Table 5: Comparison of NYHA Class, Ability Index and left/systemic ventricle echocardiographic parameters in simple CHD.

\begin{tabular}{|c|c|c|c|c|c|c|c|c|}
\hline & NYHA & Al & LVEDVI & LVESVI & LVEF & RVFunct & RVDil & PHyp \\
\hline Baseline & $1.07(0.3) 63$ & $1.0(0.1) 63$ & $57.7(14.9) 14$ & 27.9 (12.4) 11 & $61.5(6.4) 15$ & $0.1(0.2) 17$ & $0.2(0.7) 17$ & $0.2(0.7) 17$ \\
\hline $1^{\text {st }}-6^{\text {th }}$ mth & $1.3(0.4) 51$ & $1.1(0.3) 51$ & 61.9 (13.7) 8 & $31(11.2) 6$ & $62.7(8.9) 11$ & $0(0) 10$ & $1.5(1.9) 11$ & $0(0) 10$ \\
\hline $7^{\text {th }}-9^{\text {th }} \mathrm{mth}$ (delivery) & $1.1(0.5) 49$ & $1.2(0.4) 49$ & $62.3(16.8) 15$ & $26.2(12.4) 9$ & $62.3(9.3) 15$ & $0.2(0.4) 13$ & $0.5(1.4) 12$ & $0(0) 12$ \\
\hline $10^{\text {th }}-12^{\text {th }} \mathrm{mth}$ & $1(0) 33$ & $1(0) 33$ & $67(21.5) 11$ & $36.2(25.6) 11$ & $58.6(5.9) 12$ & $0(0) 12$ & $0.5(1.4) 12$ & $0(0) 12$ \\
\hline $13^{\text {th }}-24^{\text {th }} \mathrm{mth}$ & $-(-) 0$ & $-(-) 0$ & $72.8(33.3) 4$ & 34.5 (21.9) 4 & $54.8(6.6) 4$ & $0.3(0.5) 4$ & $2.3(1.9) 4$ & $0(0) 4$ \\
\hline $25^{\text {th }}-48^{\text {th }}$ mth & $-(-) 0$ & $-(-) 0$ & $132(0) 1^{*}$ & $71(0) 1^{*}$ & $46(0) 1^{*}$ & $0(0) 1^{*}$ & $5(0) 1^{*}$ & $0(0) 1^{*}$ \\
\hline
\end{tabular}

Results are expressed as mean, (SD value) and number of observations.

Al: Ability Index; LVEDVI : Left Ventricle End-Diastolic Volume Indexed ( $\left.\mathrm{mL} / \mathrm{m}^{2}\right)$; LVEF: Left Ventricle Ejection Fraction (\%); LVESVI: Left Ventricle End-Systolic Volume Indexed (mL/m²); NYHA: NYHA functional class; PHyp: Pulmonary Hypertension ( 0 = absent; 1 = present); RVDil: Right Ventricle Dilatation (0 if RVEDA 8-20 $\mathrm{cm}^{2} ; 1$ if RVEDA $21-23 \mathrm{~cm}^{2} ; 2$ if RVEDA $24-26 \mathrm{~cm}^{2} ; 3$ if RVEDA $27-29 \mathrm{~cm}^{2} ; 4$ if RVEDA > $29 \mathrm{~cm}^{2}$ ); RVEDA: Right Ventricle End-Diastolic Area (cm²); RVFunct: Right Ventricle Function $(0=$ normal; 1 = mildly impaired; $2=$ moderately impaired; $3=$ severely impaired); mth: month from last menstrual period.

* peri-operative complications

Table 6: Comparison of NYHA Class, Ability Index and left/systemic ventricle echocardiographic parameters in complex CHD. Results are expressed as mean, (SD value) and number of observations.

\begin{tabular}{|c|c|c|c|c|c|c|c|c|}
\hline & NYHA & Al & LVEDVI & LVESI & LVEF & RVFunct & RVDil & PHyp \\
\hline Baseline & $1.18(0.4) 64$ & $1.15(0.4) 64$ & $55.4(10.9) 7$ & $21.7(6.1) 6$ & $62.2(6.8) 11$ & $0.1(0.3) 12$ & $0.7(1.2) 12$ & $0.3(0.5) 14$ \\
\hline $1^{\text {st }}-6^{\text {th }}$ mth & $1.4(0.7) 53$ & $1.4(0.6) 53$ & $53.4(10.4) 5$ & $18.5(4.9) 2$ & $62.3(3.5) 10$ & $0(0) 8$ & $0.8(1.8) 8$ & $0(0) 7$ \\
\hline $7^{\text {th }}-9^{\text {th }}$ mth (del) & $1.4(0.6) 51$ & $1.4(0.6) 51$ & $58.6(14.2) 7$ & $22.5(7.2) 4$ & $60.7(5.9) 10$ & $0(0) 11$ & $2.1(2.2) 11$ & $0.2(0.4) 10$ \\
\hline $10^{\text {th }}-12^{\text {th }} \mathrm{mth}$ & $1.4(0.6) 37$ & $1.3(0.5) 37$ & $62.4(14.7) 27$ & $20.1(5.7) 9$ & $62.1(5.9) 29$ & $0.1(0.3) 30$ & 3.4 (2.1) 30 & $0.4(0.5) 28$ \\
\hline $13^{\text {th }}-24^{\text {th }} \mathrm{mth}$ & $1.6(0.7) 17$ & $1.4(0.6) 17$ & 62.9 (11.9) 20 & $22.2(5.4) 5$ & $61.0(7.3) 21$ & $0.2(0.5) 21$ & 3.3 (1.9) 21 & $0.2(0.4) 21$ \\
\hline $25^{\text {th }}-48^{\text {th }} \mathrm{mth}$ & $1.5(0.5) 8$ & $1.1(0.4) 8$ & $57.9(16.2) 16$ & $27.1(14.6) 14$ & $58(5.8) 17$ & $0.6(0.7) 16$ & $4(2.1) 15$ & $0.4(0.5) 16$ \\
\hline $49^{\text {th }}-72^{\text {th }}$ mth & $1.4(0.5) 5$ & $1.2(0.4) 5$ & 58.9 (15.2) 8 & $25.2(11.4) 6$ & $58.4(7.7) 8$ & $0.8(0.9) 8$ & $4(1.6) 8$ & $0.4(0.5) 8$ \\
\hline $73^{\text {th }}-120^{\text {th }} \mathrm{mth}$ & $2(0.7) 5$ & $1.2(0.4) 5$ & $57.8(12.2) 5$ & $27(10.8) 5$ & $53.8(8.0) 5$ & $0.8(0.8) 5$ & $3.4(2.3) 5$ & $0.6(0.5) 5$ \\
\hline $121^{\text {st }}-240^{\text {th }} \mathrm{mth}$ & $2(0) 56$ & $1.2(0.2) 9$ & $63.6(12.3) 7$ & $29.6(10.4) 7$ & $53.7(9.0) 7$ & $1.5(0.6) 4$ & $4(2) 4$ & $0.7(0.6) 3$ \\
\hline $241^{\text {st }}-360^{\text {th }} \mathrm{mth}$ & $2(0) 2$ & $1(0) 2$ & $80(0) 1$ & $38(0) 1$ & $52(0) 1$ & $1(0) 2$ & $2(1.4) 2$ & $0(0) 2$ \\
\hline
\end{tabular}

Al: Ability Index; LVEDVI : Left Ventricle End-Diastolic Volume Indexed ( $\left.\mathrm{mL} / \mathrm{m}^{2}\right)$; LVEF: Left Ventricle Ejection Fraction (\%); LVESVI: Left Ventricle End-Systolic Volume Indexed ( $\left.\mathrm{mL} / \mathrm{m}^{2}\right)$; NYHA: NYHA functional class; PHyp: Pulmonary Hypertension ( 0 = absent; 1 = present); RVDil: Right Ventricle Dilatation (0 if RVEDA 8-20 $\mathrm{cm}^{2} ; 1$ if RVEDA 21-23 $\mathrm{cm}^{2} ; 2$ if RVEDA 24-26 cm²; 3 if RVEDA 27-29 $\mathrm{cm}^{2} ; 4$ if RVEDA > $29 \mathrm{~cm}^{2}$ ); RVEDA: Right Ventricle End-Diastolic Area (cm²); RVFunct: Right Ventricle Function $(0=$ normal; 1 = mildly impaired; $2=$ moderately impaired; $3=$ severely impaired); mth: month from last menstrual period.

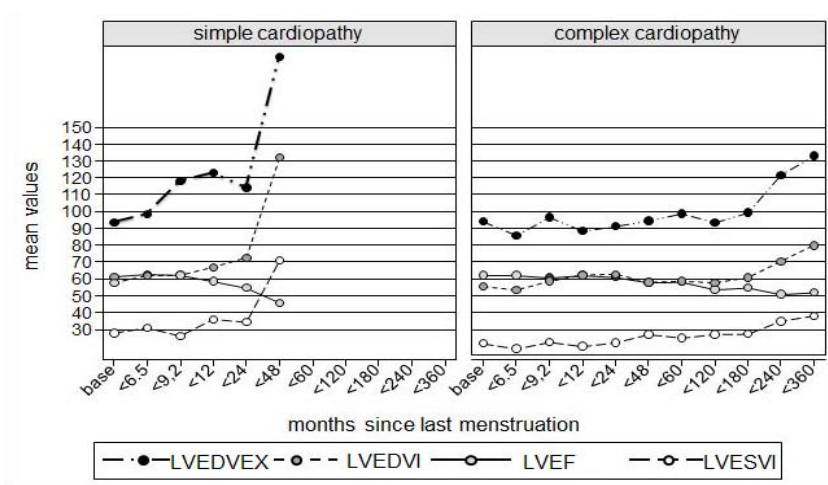

Figure 4: Echocardiographic left/systemic ventricle parameters across time. Results are shown as mean value. LVEDVEX: left ventricle end-diastolic volume $(\mathrm{mL})$; LVEDVI: Left Ventricle End-Diastolic Volume Indexed $\left(\mathrm{mL} / \mathrm{m}^{2}\right)$ LVEF left ventricle ejection fraction (\%); LVESVI Left Ventricle End-Systolic Volume Indexed $\left(\mathrm{mL} / \mathrm{m}^{2}\right)$.

a programmed preterm delivery. On the other hand, the most important factor associated with clinical worsening is with certainty the baseline NYHA Class, followed by the type of CHD (complex versus simple) and change in heart failure therapy.
Functional class index worsening occurring during pregnancy in patients with native simple $\mathrm{CHD}$ and corrected complex CHD seems to improve after the delivery, but we did not observe a complete step back to baseline values. In these patients, pregnancy may uncover structural defects, particularly of the right heart, due to the long history of heart disease or to previous surgery.

Quantitative echocardiographic data seem to support clinical findings as there is a tendency to worsening of all echocardiographic parameters during and after pregnancy, both in left and right ventricular dimension and function. Right heart parameters in complex biventricular heart CHD show the greatest worsening. This seems to be true for both simple and complex CHD, even if in long term data there could be a bias as data of simple $\mathrm{CHD}$ were available until 48 months from LMP only.

Simple CHD can be at risk during pregnancy: they are in fact silent at normal conditions but can hide a weak hemodynamic stability, uncovered by rise of blood volume and redistribution during gestation. Moreover $20 \%$ of native PFO had a TIA during pregnancy and puerperium.

Our analysis can be useful in pre-gestational counselling, because it explains in a simple way consequences of the pregnancy on mother's 


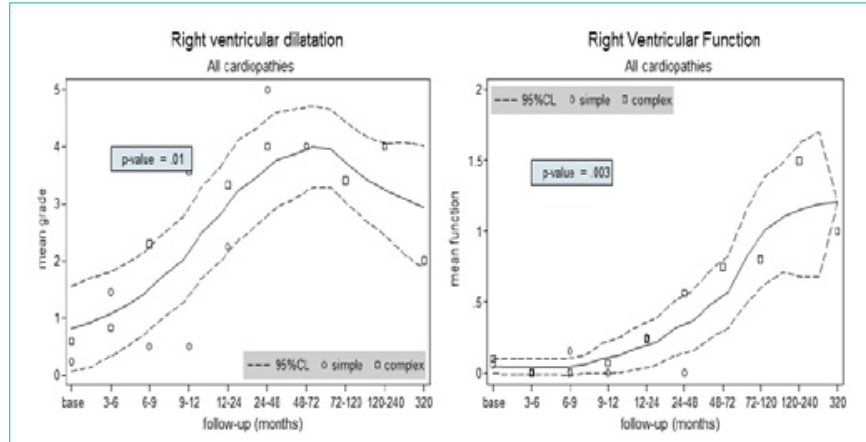

Figure 5: Echocardiographic right ventricle parameters across time.

Results are shown as mean and $95 \%$ IC. On the left: right ventricle dilatation. ( 0 if RVEDA 8-20 cm²; 1 if RVEDA $21-23 \mathrm{~cm}^{2} ; 2$ if RVEDA $24-26 \mathrm{~cm}^{2} ; 3$ if RVEDA $27-29 \mathrm{~cm}^{2} ; 4$ if RVEDA $>29 \mathrm{~cm}^{2}$ ). On the right: right ventricle function $(0=$ normal; $1=$ mildly impaired; $2=$ moderately impaired; $3=$ severely impaired). Simple: simple congenital heart disease; Complex: complex heart disease. Months refer to last menstrual period.

health in the short- and early mid- term.

NYHA Class and Ability Index are somehow complementary, and functional worsening seems to be, for both parameters, associated with the same factors. However, Ability Index change is not associated with the type of CHD: this suggests that even if women with complex CHD have more relevant underlying anomalies, they may not notice any worsening during pregnancy, as CHD already limited their life activities. About half of our patients were in cardiac therapy before the pregnancy and more than $30 \%$ began or increased it during the gestation; therapy introduction was associated with improvement of functional class after the delivery.

Functional class does not seem to be worsened by extra cardiac events during pregnancy: in fact, in our population we did not observe higher incidence of gestational hypertension (20.27\% against $15-25 \%$ in the general population), gestational diabetes $(5.4 \%$ vs. $15 \%)$ or oligohydramnios ( $2.7 \%$ vs. $2-5 \%$ ), and we observed a lower incidence of preeclampsia ( $4 \%$ vs. $8 \%$ ) and eclampsia ( 0 vs. 1 in 1000 5000 pregnancies).

Finally, the recurrence of CHD in offspring was greater in our population than in the general population, even if the CHD of the newborns was not the same as the mother's, according to previous studies $[3,19]$.

\section{Study Limitations}

This is a single centre retrospective, with possible information bias. NYHA class may be subjective, although it represents a simple index that can be easily used during routinely outpatient clinic evaluation.

Cardiac ultrasound is an operator-dependent diagnostic exam, even if all exams were performed by accredited operators. Low number of records may have prevented reach of statistical significance.

\section{Conclusions}

Pregnancy is not contraindicated in GUCH women, even in presence of complex CHD. The probability of functional class worsening during and after pregnancy can be predicted especially according to baseline NYHA Class. This is paramount during pregestational counselling and may help preventing further cardiac deterioration in pregnant women with CHD.

\section{Acknowledgements}

We sincerely thank our colleagues belonging to Department of Maternal and Pediatric Health, University of Padua, Padua, Italy who allowed data collection.

\section{References}

1. Ouzounian JG, Masaki DI, Abboud TK, Greenspoon JS. Systemic vascular resistance index determined by thoracic electrical bioimpedance predicts the risk for maternal hypotension during regional anesthesia for cesarean delivery. Am J Obstet Gynecol [Internet]. 1996; 174: 1019-1025.

2. Khairy $\mathrm{P}$, Ionescu-Ittu R, Mackie AS, Abrahamowicz M, Pilote L, Marelli AJ. Changing mortality in congenital heart disease. J Am Coll Cardiol [Internet]. 2010; 56: 1149-1157.

3. Presbitero P, Somerville J, Stone S, Aruta E, Spiegelhalter D, Rabajoli F. Pregnancy in cyanotic congenital heart disease. Outcome of mother and fetus. Circulation [Internet]. 1994; 89: 2673-2676.

4. Drenthen W, Pieper PG, Roos-Hesselink JW, van Lottum WA, Voors AA, Mulder BJM, et al. Outcome of pregnancy in women with congenital heart disease: a literature review. J Am Coll Cardiol [Internet]. 2007; 49: 23032311.

5. Drenthen W, Boersma E, Balci A, Moons P, Roos-Hesselink JW, Mulder BJM, et al. Predictors of pregnancy complications in women with congenital heart disease. Eur Heart J [Internet]. 2010; 31: 2124-2132.

6. European Society of Gynecology (ESG), Association for European Paediatric Cardiology (AEPC), German Society for Gender Medicine (DGesGM), Regitz-Zagrosek V, Blomstrom Lundqvist C, Borghi C, et al. ESC Guidelines on the management of cardiovascular diseases during pregnancy: the Task Force on the Management of Cardiovascular Diseases during Pregnancy of the European Society of Cardiology (ESC). Eur Heart J [Internet]. 2011; 32: 3147-3197.

7. Siu SC, Sermer M, Colman JM, Alvarez AN, Mercier LA, Morton BC, et al. Prospective multicenter study of pregnancy outcomes in women with heart disease. Circulation [Internet]. 2001; 104: 515-521.

8. Khairy P, Ouyang DW, Fernandes SM, Lee-Parritz A, Economy KE, Landzberg MJ. Pregnancy outcomes in women with congenital heart disease. Circulation [Internet]. 2006; 113: 517-524.

9. Warnes CA, Somerville J. Tricuspid atresia in adolescents and adults: current state and late complications. Br Heart J [Internet]. 1986; 56: 535-543.

10. Lang RM, Badano LP, Mor-Avi V, Afilalo J, Armstrong A, Ernande L, et al. Recommendations for cardiac chamber quantification by echocardiography in adults: an update from the American Society of Echocardiography and the European Association of Cardiovascular Imaging. J Am Soc Echocardiogr Off Publ Am Soc Echocardiogr [Internet]. 2015; 28: 1-39.e14

11. Apgar V. A proposal for a new method of evaluation of the newborn infant. Curr Res Anesth Analg [Internet]. 1953; 32: 260-267.

12. Royston P, Lambert PC. Flexible Parametric Survival Analysis Using Stata: Beyond the Cox Model. Stata Press. 2011.

13. Balci A, Sollie-Szarynska KM, van der Bijl AGL, Ruys TPE, Mulder BJM, RoosHesselink JW, et al. Prospective validation and assessment of cardiovascular and offspring risk models for pregnant women with congenital heart disease. Heart [Internet]. 2014; 100: 1373-1381.

14. Regitz-Zagrosek V, Blomstrom Lundqvist C, Borghi C, Cifkova R, Ferreira $\mathrm{R}$, Foidart JM, et al. ESC Guidelines on the management of cardiovascular diseases during pregnancy. Eur Heart J. 2011; 32: 3147-3197.

15. Gelson E, Curry R, Gatzoulis MA, Swan L, Lupton M, Steer P, et al. Effect of maternal heart disease on fetal growth. Obstet Gynecol [Internet]. 2011; 117: 886-891. 
16. Uebing A, Arvanitis P, Li W, Diller GP, Babu-Narayan SV, Okonko D, et al Effect of pregnancy on clinical status and ventricular function in women with heart disease. Int J Cardiol [Internet]. 2010; 139: 50-59.

17. Daliento L, Dal Bianco L, Bagato F, Secco E, Sarubbi B, Mazzotti E, et al. Gender differences and role of pregnancy in the history of post-surgical women affected by tetralogy of Fallot. PLoS One [Internet]. 2012; 7: e49729.

18. Norozi K, Wessel A, Buchhorn R, Alpers V, Arnhold JO, Zoege M, et al. Is the Ability index superior to the NYHA classification for assessing heart failure?: comparison of two classification scales in adolescents and adults with operated congenital heart defects. Clin Res Cardiol Off J Ger Card Soc [Internet]. 2007; 96: 542-547.

19. Burn J, Brennan P, Little J, Holloway S, Coffey R, Somerville J, et al. Recurrence risks in offspring of adults with major heart defects: results from first cohort of British collaborative study. Lancet (London, England) [Internet] 1998; 351: 311-316.
J Cardiovasc Disord - Volume 4 Issue 1 - 2017

ISSN 2379-7991 | www.austinpublishinggroup.com

Daliento et al. (C) All rights are reserved
Citation: Daliento L, Pomiato E, Mazzotti E, Bauce B, Spadotto V and Rizzoli G. Pregnancy-Induced Functional Class Modifications in Patients with Congenital Heart Disease. J Cardiovasc Disord. 2017; 4(1): 1032. 